\title{
Vapor Deposition of Ruthenium from an Amidinate Precursor
}

\author{
Huazhi Li, ${ }^{\mathrm{a}}$ Damon B. Farmer, ${ }^{\mathrm{b}}$ Roy G. Gordon ${ }^{*},{ }^{\mathrm{a}}$ Youbo Lin, ${ }^{\mathrm{b}}$ Joost Vlassak ${ }^{\mathrm{b}}$ \\ ${ }^{a}$ Department of Chemistry and Chemical Biology and ${ }^{b}$ School of Engineering and Applied Sciences, \\ Harvard University, Cambridge, Massachusetts 02138, USA
}

\begin{abstract}
Atomic layer deposition (ALD) and pulsed chemical vapor deposition (CVD) were used to make ruthenium $(\mathrm{Ru})$ thin films from a volatile $\mathrm{Ru}$ amidinate precursor, bis( $N, N$ '-di-tert-butylacetamidinato)ruthenium(II) dicarbonyl. The CVD films were grown without any co-reactant, while the ALD films used ammonia as a co-reactant. The films are fine-grained polycrystalline ruthenium with high purity ( $<0.2 \%$ impurities). $\mathrm{Ru}$ grew as a continuous, electrically conductive, pinhole-free film on WN films even for films as thin as $2 \mathrm{~nm}$. The resistivities of the films match those of pure sputtered ruthenium of the same thickness. Roughness is less than $2 \%$ of the film thickness. The films are very conformal, with $80 \%$ step coverage over holes with high aspect ratios (40:1). This thermal process does not use any oxidant or plasma as a second reagent, thereby avoiding damage to sensitive substrates. The ALD growth rate can reach 1.5 $\AA$ /cycle at a substrate temperature of $300{ }^{\circ} \mathrm{C}$.
\end{abstract}

Key words: ruthenium, adhesion layer, ALD, thin film.

* To whom correspondence should be addressed. E-mail: gordon@chemistry.harvard.edu 


\section{Introduction}

Thin films of ruthenium have many current and potential applications. They can be used as electrodes for capacitors, in which their high work function leads to low leakage currents. ${ }^{1}$ Furthermore, the conductive nature of $\mathrm{RuO}_{2}$ interlayers with oxide insulators promotes high capacitance density. As gate electrodes, the high work function of Ru provides low threshold voltages for PMOS transistors. ${ }^{2,3}$ Because Ru has a low solid solubility in copper and strong adhesion to copper, it could be used as a seed and/or

adhesion layer for copper interconnects. ${ }^{4}$ Multilayer magnetoresistance structures such as those found in read/write heads in hard-drives and in magnetic memory (MRAM) can use $\mathrm{Ru}$ as a non-magnetic spacer between magnetic layers. ${ }^{5}$ Some plasma displays incorporate Ru films. ${ }^{6}$ Heterogeneous catalysts can be made from Ru films deposited on a support. ${ }^{7}$

Physical vapor deposition (PVD) methods such as sputtering can be used to deposit ruthenium films. Due to the directional nature of the deposition flux and the high sticking probability, PVD technology cannot deposit ruthenium inside narrow holes and complex structures. Ru films with better conformity are sometimes achieved by using chemical vapor deposition (CVD). Atomic layer deposition (ALD) can make completely conformal coating even in structures with high aspect ratios.

Many CVD Ru processes have been reported. Precursors based on the cyclopentadienyl $(\mathrm{Cp})$ ligands such as $\mathrm{RuCp}_{2}$ and $\mathrm{Ru}(\mathrm{EtCp})_{2}(\mathrm{Et}=$ ethyl $)$ have been investigated. ${ }^{8}$ Ruthenium tris- $\beta$-diketonates were also widely studied in CVD ruthenium and ruthenium oxide films. ${ }^{9}$ These include $\mathrm{Ru}(\mathrm{acac})_{3}$ (acac $=2,4$-pentanedionate), $\mathrm{Ru}$ (thd) $)_{3}$ (thd = 2,2,6,6-tetramethyl-3,5-heptanedionate), Ru(tfa) ${ }_{3}$ (tfa = 1,1,1-trifluoro- 
2,4-pentanedionate), and $\mathrm{Ru}(\mathrm{OD})_{3}\left(\mathrm{OD}=2\right.$,4-octanedionate). $\mathrm{Ru}_{3}(\mathrm{CO})_{12}, \mathrm{Ru}(\mathrm{CO})_{4}(\mathrm{hfb})$

(hfb = hexafluoro-2-butyne), $\mathrm{Ru}(\mathrm{CO})_{2}(\mathrm{hfac})_{2}(\mathrm{hfac}=1,1,1,5,5,5$-hexafluoro-2,4-

pentanedionate), and $\mathrm{Ru}(\mathrm{CO})_{2}(\text { thd })_{2}$ were also tried as CVD Ru precursors. ${ }^{10}$ However, these precursors either lack thermal stability, do not produce pure films and/or fail to provide high step coverage.

Film growth in ALD is based on alternating, self-limited surface reactions. When precursors show this self-limiting growth mechanism, the films deposited by ALD have excellent conformality and superior uniformity. ALD Ru based on precursors RuCp $\mathrm{p}_{2},{ }^{11}$ $\mathrm{Ru}(\mathrm{EtCP})_{2},{ }^{12} \mathrm{Ru}(\mathrm{thd}),{ }^{13}$ and $\mathrm{Ru}(\mathrm{OD})_{3}{ }^{14}$ have been the subject of recent studies. However, all of these processes require using $\mathrm{O}_{2}$ or ozone as a second reagent, which can potentially form interfacial metal oxide films that can cause interconnect failure or increase contact resistance. $\mathrm{NH}_{3}$ plasma has also been tried as the second reagent to avoid the formation of interfacial metal oxides, but a plasma may not achieve high step coverage because of rapid recombination of plasma-generated radicals on the surfaces of features. ${ }^{15}$ Also, plasma reactors are more complicated than thermal reactors, and plasmas can damage substrates.

Here we present a vapor deposition process for ruthenium using bis( $N, N^{\prime}$-di-tertbutylacetamidinato)ruthenium(II) dicarbonyl, Ru( $\left.{ }^{t} \mathrm{Bu}-\mathrm{Me}-\mathrm{amd}\right)_{2}(\mathrm{CO})_{2} \mathbf{1}$, as shown in Fig. 1. This precursor can be used alone in a pulsed CVD mode or with reducing agents such as molecular hydrogen or ammonia in a mixed CVD-ALD mode. The processes are thermally activated, and do not require the use of oxygen, ozone or plasmas.

\section{Results and Discussion}




\section{Film Growth}

Pulsed CVD consisted of (a) supplying a dose of Ru precursor vapor 1 (bis $\left(N, N^{\prime}\right.$ di-tert-butylacetamidinato)ruthenium(II) dicarbonyl) mixed with $\mathrm{N}_{2}$ carrier gas to a uniformly heated chamber containing substrates; (b) purging the deposition chamber with a vacuum pump and the assistance of a dose of pure $\mathrm{N}_{2}$ gas. Figure 2 shows the pulsed thermal CVD growth of Ru from precursor 1 on thermal $\mathrm{SiO}_{2}$ substrates. Above $200{ }^{\circ} \mathrm{C}$ there was substantial deposition, while below $200{ }^{\circ} \mathrm{C}$ there was little or no deposition. At $300{ }^{\circ} \mathrm{C}$, the observed CVD rate was around $0.4 \AA$ /pulse. Figure 3 shows the Arrhenius representation of the CVD rate as a function of the substrate temperature for the CVD process. Over a wide range of temperatures $\left(200-300^{\circ} \mathrm{C}\right)$, the curve is linear, which means that the CVD rate is kinetically controlled in that temperature range. From these data, the activation energy of the CVD process was calculated to be $81 \mathrm{~kJ} / \mathrm{mol}$.

$\mathrm{NH}_{3}$ is an optional co-reactant, but using $\mathrm{NH}_{3}$ can boost the growth rate of $\mathrm{Ru}$ films as shown in Figures 2 and 3. A typical ALD cycle was composed of four separate steps: (a) one dose of $\mathbf{1}$ using $\mathrm{N}_{2}$ injected into the headspace of the bubbler to assist the transport of the precursor vapor into the reactor; (b) $\mathrm{N}_{2}$ purge; (c) one dose of $\mathrm{NH}_{3}$; (d) $\mathrm{N}_{2}$ purge. Figure 2 demonstrates that in the ALD process with $\mathrm{NH}_{3}$ as a second reagent, the deposition rate is somewhat higher than with pulsed CVD (self decomposition) of the precursor without any co-reactant. The ALD growth per cycle was further increased to 1.5 Angstroms per cycle by increasing size of the vapor doses, by using a bubbler with a larger head-space and containing more precursor. Under these optimized conditions, the vapor concentration in the reactor increases and thus the pulsed CVD pathway provides 
more growth. In other words, the ALD growth is not self-limiting because it contains a CVD pathway as well as a self-limiting ALD reaction.

Because the decomposition of the precursor on the surface is slow, the film thickness can still be uniform and conformal if the temperature of the substrate is uniform, even though the growth is not self-limiting. The thickness non-uniformity of films over a $25 \mathrm{~cm}$-long deposition zone was less than $5 \%$ at $300{ }^{\circ} \mathrm{C}$. Above $300{ }^{\circ} \mathrm{C}$, the thickness non-uniformity was higher than $5 \%\left(\sim 15 \%\right.$ at $\left.325^{\circ} \mathrm{C}\right)$. The less uniform thickness at higher temperatures may be due to the surface reactions becoming so fast that the growth is limited by transport in the vapor, rather than by surface reactions. As a result, $300{ }^{\circ} \mathrm{C}$ was used as the optimized growth temperature.

A proposed mechanism of the thermal decomposition is shown in Equation 1. Here, the ruthenium precursor decomposes by releasing carbon monoxide and the ligand dimer 2, which joins at the $\mathrm{N}$ atoms while giving off two electrons to reduce the adjacent $\mathrm{Ru}(\mathrm{II})$ to $\mathrm{Ru}(0)$. This ligand dimer was isolated and the structure 2 illustrated in Equation 1 was confirmed by ${ }^{1} \mathrm{H}$ NMR as well as high-resolution mass spectroscopy and elemental analysis. Additional evidence supporting this mechanism was acquired by using carbon monoxide as the carrier gas during deposition. When this was done, the Ru deposition rate was decreased by almost half, which means that the leaving of carbon monoxide is reversible, and is relevant to the kinetics of the deposition reaction.

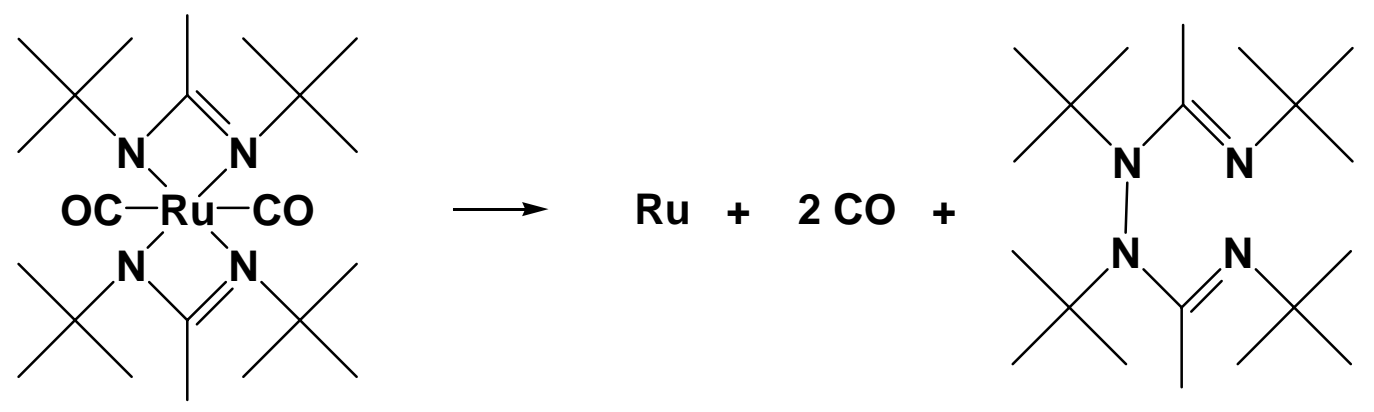


The mechanism by which the $\mathrm{NH}_{3}$ enhances the ALD deposition rate is not known.

\section{Film Properties}

RBS analyses of films on glassy carbon substrates show no evidence of carbon, nitrogen or oxygen in either the CVD or ALD films, which indicates that $\mathrm{NH}_{3}$ molecules are not incorporated in the films (data not shown here). The lack of nitrogen incorporation is not surprising, because ruthenium does not have any stable nitride phase. Secondary ion mass spectra (SIMS) in Figure 4 show that the impurity content of the ruthenium films grown at $300{ }^{\circ} \mathrm{C}$ is very low. The carbon content in the films is around $0.05 \%$ while the oxygen content is around $0.2 \%$. These results verify that the surface reactions are fully complete under the conditions used, and that ruthenium is not oxidized during the deposition process or in the ambient environment.

Atomic force microscopy (AFM) was employed to study the surface roughness of the as-deposited Ru thin films. Figure 5 shows an AFM image of the film morphology. The root mean square (rms) roughness is $1.5 \mathrm{~nm}$ for an 87-nm-thick film deposited at 300 ${ }^{\circ} \mathrm{C}$, which corresponds to a fairly smooth film.

Examination of thicker films by XRD showed that they are polycrystalline ruthenium films. Figure 6 shows X-ray diffraction spectra measured from a 180-nmthick film deposited at $300^{\circ} \mathrm{C}$. These films were randomly oriented. Annealing these films at $500{ }^{\circ} \mathrm{C}$ and $750{ }^{\circ} \mathrm{C}$ in $\mathrm{N}_{2}$ at 15 Torr for $1 \mathrm{~min}$ did not cause the XRD spectrum to change significantly, indicating that Ru recrystallization is negligible at these temperatures.

The TEM image of a 30-nm-thick film on a $\mathrm{Si}_{3} \mathrm{~N}_{4}$ membrane shows that the grain size is about 5 to $20 \mathrm{~nm}$ (Figure 7). Because the film was deposited on both sides of the 
membrane, some Moire patterns appear due to interference between grains in the two films. These investigations lead to the conclusions that the Ru films are smooth, nanocrystalline, and structurally stable.

A test of film continuity was made by depositing a 2-nm-thick Ru film on a $3 \mathrm{~nm}$ thick freshly deposited tungsten nitride film on a $30 \mathrm{~nm} \mathrm{Si}_{3} \mathrm{~N}_{4}$ membrane. The TEM of this structure in Figure 8 shows that the Ru grains on WN are much smaller and more densely spaced than on $\mathrm{Si}_{3} \mathrm{~N}_{4}$ (Figure 7). This sample was then submerged in a WN etching solution $\left[\mathrm{H}_{2} \mathrm{O}_{2}: \mathrm{NH}_{3}: \mathrm{H}_{2} \mathrm{O}(1: 1: 5)\right]$ for 10 mins. In a separate experiment, this solution was found not to etch Ru films. Energy dispersive X-ray spectroscopy (EDX) analysis revealed the presence of Ru and $\mathrm{W}$ both before and after submersion in the etchant. In contrast, a WN film that was not capped with a $2 \mathrm{~nm}$ Ru layer was completely etched in the same solution within seconds. These results show that there are no gaps or pinholes in this very thin Ru film. TEM analysis of the Ru-capped film before and after etching showed no difference in terms of the size or density of nuclei.

To extend on these results, the layers were intentionally pierced with holes and then left in the etching solution for $\sim 12$ hours to ensure complete dissolution of the WN by undercutting from the edges of the holes. After etching, some fragments of the Ru film settled back onto the substrate. Figure 9 shows a TEM image of one of these pieces of Ru film that had been released from the substrate by dissolution of the WN. The Ru film is clearly continuous, free of pin-holes and self-supporting. The existence of these intact fragments is further proof of the continuity and uniformity of the 2-nm-thick Ru films. 
The in-plane resistivities of the Ru films on WN were measured with a 4-point probe. A small correction was made for the parallel conduction through the WN. The Ru resistivity increases with decreasing film thickness owing to several effects: grain boundary scattering, electron-surface scattering, surface-roughness-induced scattering, and impurity scattering. The curve in Figure 10 is calculated from a simple model that includes only grain-boundary scattering, ${ }^{16}$

$$
\rho / \rho_{0}=1+1.5\{R /(1-R)\}(\lambda / g)
$$

where $\rho_{0}$ is the bulk resistance of $7.1 \mu \Omega-\mathrm{cm}, \lambda$ is the electron mean free path (estimated to be $10 \mathrm{~nm}), g$ is the average size of the grains (approximated as the film thickness), and $R \sim 0.58$ is the grain-boundary-scattering coefficient (obtained by fitting the data in Fig. 10). It is remarkable that the data for Ru films produced by ALD follow this model down to thicknesses of $2 \mathrm{~nm}$. This agreement between measured conductivity and the model suggests that the ruthenium film must be continuous and nearly uniformly covering the WN surface. The agreement between the resistivity of the ALD Ru films and pure sputtered Ru films shows that impurities in the ALD films do not have any significant effect on their electrical resistance.

One of the challenges in multilevel metallization of the sub-90 $\mathrm{nm}$ generation devices is the void-free filling of high aspect ratio contact holes. Non-conformal Ru films can cause shoulders near the top of the holes, leading to void formation when the holes are filled by $\mathrm{Cu}$ or $\mathrm{W}$. The CVD and ALD Ru films were highly conformal inside holes in Si with aspect ratios of 40:1. Figure 11 shows a scanning electron microscopy (SEM) image of cleaved cross sections of ALD Ru films inside UV/ozone treated Si holes. The film thickness at the top of the holes is found to be $50 \mathrm{~nm}$, while the thickness at the 
middle and bottom of the holes is $40 \mathrm{~nm}$. This gives a step coverage of $80 \%$. The deviation away from ideal $100 \%$ step coverage may be due to the partial self decomposition of the precursor, or to insufficient exposure to the precursor. ${ }^{17}$

$\mathrm{Ru}$ adhesion energies are listed in Table I. The $\mathrm{Ru} / \mathrm{SiO}_{2}$ interface can be separated by Scotch tape. Four point bend tests for $\mathrm{Ru} / \mathrm{SiO}_{2}$ give an adhesion energy of less than $3 \mathrm{~J} \mathrm{~m}^{-2}{ }^{18}$ In contrast, the $\mathrm{Ru} / \mathrm{WN} / \mathrm{SiO}_{2}$ stack passes the tape test and exhibits strong adhesion. Four point bend tests indicate that the $\mathrm{WN} / \mathrm{SiO}_{2}$ interface has an adhesion energy of at least $17.6 \pm 3.8 \mathrm{~J} / \mathrm{m}^{2}$, where the location of the adhesive failure was confirmed by XPS analysis of both interfaces exposed by the crack. It was not possible to break the $\mathrm{Ru} / \mathrm{WN}$ interface in the film stack under the same testing conditions.

\section{Conclusions}

We conclude that CVD and ALD of Ru on WN can provide highly conformal, continuous, and strongly adherent layers that are suitable for many applications. The process does not require oxidizing conditions or plasmas, which can damage substrates. The Ru films nucleate densely on freshly-deposited WN and are continuous and pin-holefree at thicknesses down to $2 \mathrm{~nm}$. The polycrystalline films are substantially free of impurities, and have resistivities matching those of pure sputtered Ru films.

\section{Acknowledgments}

We appreciate the help with the AFM imaging from Dr. Zhengwen Li and Qiaobing Xu. Youbo Lin acknowledges support from SRC, contract 2005-KC-1292.010. A portion of the work presented here was carried out at the Center for Nanoscale Systems at Harvard University. This work was supported in part by the National Science Foundation. 


\section{Experimental}

Pulsed CVD of ruthenium was carried out by thermal decomposition of bis $\left(N, N^{\prime}-\right.$ di-tert-butylacetamidinato) ruthenium(II) dicarbonyl, $\mathrm{Ru}\left({ }^{\mathrm{t}} \mathrm{Bu}-\mathrm{Me} \text {-amd }\right)_{2}(\mathrm{CO})_{2}$ without any other reactant or reducing agent. The detailed synthesis and characterization of the precursor will be published separately. ${ }^{19}$ The compound is monomeric, with a distorted octahedral structure. The vapor pressure of $\mathrm{Ru}\left({ }^{\mathrm{t}} \mathrm{Bu}-\mathrm{Me}-\mathrm{amd}\right)_{2}(\mathrm{CO})_{2}$ is about 0.05 Torr at $130{ }^{\circ} \mathrm{C}$, based on sublimation data. 10 grams of the Ru precursor were placed in a stainless steel container with a volume of $130 \mathrm{~cm}^{3}$ and heated (typically to a temperature of $\left.130{ }^{\circ} \mathrm{C}\right)$ in an oven. Nitrogen carrier gas $\left(2.5 \times 10^{-4}\right.$ moles) was admitted to the headspace volume above the precursor, resulting in a gas mixture of $1 \mathrm{~mol} \% \mathrm{Ru}$ precursor and $99 \mathrm{~mol} \% \mathrm{~N}_{2}$ at a total pressure of about 6.4 mbar. Meanwhile, the deposition chamber (a stainless steel tube with volume $386 \mathrm{~cm}^{3}$ ) in a tube furnace was pumped down to a base pressure of about $0.05 \mathrm{mbar}$, and then an air-actuated valve between the deposition chamber and the pump was closed, admitting a dose of $1.8 \times 10^{-6}$ moles of Ru precursor to the deposition chamber. The coated area (including the substrates, the substrate holder and the heated chamber walls) was $686 \mathrm{~cm}^{2}$, so the dose was 0.26 nanomoles per square centimeter. The valve between the precursor source and the deposition chamber was next opened for 2 seconds and then closed. After a period of 2 more seconds, the valve between the deposition chamber and the pump was opened for 25 seconds, and $5.6 \times 10^{-4}$ moles of nitrogen purging gas was admitted to help sweep out byproducts and any unreacted precursor vapor. The exposure of the Ru precursor to the surfaces was 0.086 mbar-sec. This CVD dose was then repeated a specified number of times. 
ALD of ruthenium was carried out with the same equipment as was used for the pulsed CVD of Ru. The doses of Ru precursor vapor were alternated with doses of ammonia gas. The ammonia dose from a measured volume $\left(22 \mathrm{~cm}^{3}\right.$ at a pressure of 1.24 bar) flowed into the evacuated deposition region, where it was also confined for a time period (typically 3 seconds) before purging. The dose of ammonia was 1.06 micromole $\mathrm{cm}^{-2}$ and the exposure was 200 mbar-sec.

The substrates used had $300 \mathrm{~nm}$ of thermal oxide on Si (100). Before deposition, the substrates were treated for 4 minutes with UV/ozone to remove organic contaminants. ALD WN as a diffusion barrier and adhesion layer was deposited on the $\mathrm{SiO}_{2}$ substrates at $380{ }^{\circ} \mathrm{C}$ using ALD with bis(tert-butylimido)bis(dimethylamido) tungsten(VI) and ammonia gas as precursors. ${ }^{20}$ Typically, 10 cycles were used to make a WN layer about $2.5 \mathrm{~nm}$ thick.

Some Ru samples were annealed in a rapid thermal annealing (RTA) furnace at $500{ }^{\circ} \mathrm{C}$ and $750{ }^{\circ} \mathrm{C}$ for $1 \mathrm{~min}$ in a vacuum (14.7 Torr). The crystal orientation of the asdeposited and annealed Ru films was evaluated by using a $\theta-2 \theta$ x-ray diffractometer (XRD; Scintag. Inc., USA) with $\mathrm{Cu} \mathrm{K}_{\alpha}$ radiation generated at $40 \mathrm{kV}$ and $30 \mathrm{~mA}$. Film resistivity was calculated from the sheet resistance measured with a four-point probe and the film thickness. The film thickness was measured using $2 \mathrm{MeV} \mathrm{He}^{+}$Rutherford backscattering spectroscopy (RBS). TEM pictures were taken with Ru/WN stacks on amorphous $\mathrm{SiO}_{2}$ or $\mathrm{Si}_{3} \mathrm{~N}_{4}$ membrane grids supplied by SPI Supplies.

Adhesion measurements were performed using the four point bend technique. Test specimens were prepared by bonding Si wafers to the substrates with the $\mathrm{Ru} / \mathrm{WN} / \mathrm{SiO}_{2}$ film stack using a spin-on epoxy (EPOTEK353ND). The epoxy was cured 
at $90^{\circ} \mathrm{C}$ for 60 min under a pressure of $8 \mathrm{kPa}$. The epoxy thickness after the curing cycle was approximately $5 \mu \mathrm{m}$. Prior to spin-coating the epoxy, $40 \mathrm{~nm}$ of Ti and $400 \mathrm{~nm}$ of $\mathrm{Cu}$ were sputter deposited onto the Ru to increase the distance between the film stack and the epoxy. All four point bend tests were performed in dry $\mathrm{N}_{2}$ at $25^{\circ} \mathrm{C}$ at a crosshead speed of $0.3 \mu \mathrm{m} / \mathrm{s}$. All experimental loading curves displayed well-developed load plateaus during crack extension allowing the determination of the adhesion energy. ${ }^{21}$ 
Table I. Adhesion measurements for ALD and CVD multilayer structures. Adhesion energy was evaluated by four-point bend tests.

Adhesion energy

Structure

Scotch tape test

$\left(\mathrm{J} / \mathrm{m}^{2}\right)$

$\overline{\mathrm{Ru} / \mathrm{SiO}_{2}}$

Failed

$<3$

$\mathrm{WN} / \mathrm{SiO}_{2}$

Passed

$17.6 \pm 3.8 \mathrm{~J} / \mathrm{m}^{2}$

$\mathrm{Ru} / \mathrm{WN} / \mathrm{SiO}_{2}$

Passed

$>17.6 \mathrm{~J} / \mathrm{m}^{2}$ 


\section{Figure Captions}

Figure 1. (a) The chemical formula of Ru precursor bis( $N, N^{\prime}$-di-tertbutylacetamidinato)ruthenium(II) dicarbonyl, Ru( $\left.{ }^{\mathrm{B}} \mathrm{Bu}-\mathrm{Me}-\mathrm{amd}\right)_{2}(\mathrm{CO})_{2}$.

(b) its molecular structure by X-ray crystallography (hydrogen atoms not shown).

Figure 2. The Ru growth rate with $\mathrm{NH}_{3}$ (ALD) or without $\mathrm{NH}_{3}$ (pulsed CVD) at different temperatures.

Figure 3. Pulsed CVD growth rate of Ru without using $\mathrm{NH}_{3}$ (squares) and ALD growth rate (circles) as a function of temperature.

Figure 4. SIMS depth profiles of a ruthenium film $20 \mathrm{~nm}$ thick deposited on thermal $\mathrm{SiO}_{2}$ at $300{ }^{\circ} \mathrm{C}$; the observed carbon and oxygen counts/second have been converted to concentrations; the ruthenium curve represents the signal in counts per second because a sensitivity factor is not known for Ru.

Figure 5. AFM image of a $87 \mathrm{~nm}$ ruthenium film grown at $300^{\circ} \mathrm{C}$. (a) 2-D view; (b) 3-D view.

Figure 6. XRD patterns obtained from a Ru film as deposited and after annealing.

Figure 7. TEM images of a Ru film (30 nm) deposited over $\mathrm{Si}_{3} \mathrm{~N}_{4}$ membrane (30nm). 
Figure 8. TEM of a Ru film grown on freshly deposited tungsten nitride on a $\mathrm{Si}_{3} \mathrm{~N}_{4} \mathrm{TEM}$ membrane.

Figure 9. TEM image of a fragment of a Ru film of after etching of WN in a pierced film of $\mathrm{Ru}(2 \mathrm{~nm}) / \mathrm{WN}(3 \mathrm{~nm}) / \mathrm{SiO}_{2}$ deposited on $\mathrm{Si}_{3} \mathrm{~N}_{4}$ membrane (30 nm). The darker areas are regions where the film folded over on itself.

Figure 10. The resistivity of Ru thin films as a function of Ru film thickness on WN substrates. Black squares are data from this study, gray dots are data from sputtered Ru.

Figure 11. Ru films deposited inside the high aspect ratio (40:1) trench structure showing excellent conformality of pulsed CVD process. The white bar on the bottom of each image represents $200 \mathrm{~nm}$. 


\section{References}

${ }^{1}$ a) C. S. Hwang, Mater. Sci. Eng. B 56, 178 (1998); b) M. Nayak, S. Ezhilvalavan, T. Y. Tseng, in Handbook of Thin Film Materials, H. S. Nalwa, Ed., Academic Press, San Diego, CA, p.121 (2001).

${ }^{2}$ M. Tapajna, P. Pisecny, R. Luptak, K. Husekova, K. Froehlich, L. Harmatha, J. C.

Hooker, F. Roozeboom, J. Jergel, Materials Science in Semiconductor Processing 7, 271 (2004).

${ }^{3}$ T. Seidel, A. Londergan, J. Winkler, X. Liu, S. Ramanathan, Solid State Technol. 46, 67 (2003).

${ }^{4}$ I. Goswami, R. Laxman, Semiconductor International 27, 49 (2004).

${ }^{5}$ T. Takenaga, T. Kuroiwa, T. Furukawa, M. Taki, K. Yoshiara, Y. Tokuda, Jpn. J. Appl. Phys. 99, 08C911 (2006).

${ }^{6}$ B. J. Mun and S. Hak, US Patent 7,125,655.

${ }^{7}$ A. Koeckritz, M. Sebek, A. Dittmar, J. Radnik, A. Brueckner, U. Bentrup, M.-M. Pohl, H. Hugl, W. Maegerlein, J. Molecular Catalysis A: Chemical 246, 85 (2006).

${ }^{8}$ M. L. Green, M. E. Gross, L. E. Papa, K. J. Schnoes, and D. Brasen, J. Electrochem. Soc. 132, 2677 (1985); T. Aoyama and K. Eguchi, Jpn. J. Appl. Phys. 38, L1134 (1999).

${ }^{9}$ J. C. Viguié and J. Spitz, J. Electrochem. Soc. 122, 585 (1975); J. Vetrone, C. M. Foster, G.-R. Bai, A. Wang, J. Patel, and X. Wu, J. Mater. Res. 13, 2281 (1998); J.-H. Lee, J.-Y. Kim, S.-W. Rhee, D. Y. Yang, D.-H. Kim, C.-H. Yang, Y.-K. Han, and C.-J. Hwang, J. Vac. Sci. Technol. A 18, 2400 (2000).

${ }^{10}$ E. P. Boyd, D. R. Ketchum, H. Deng, and S. G. Shore, Chem. Mater. 9, 1154 (1997); Y. Senzaki, W. L. Gladfelter, and F. B. McCormick, Chem. Mater. 5, 1715 (1993); Y.-H. 
Lai, Y.-L. Chen, Y. Chi, C.-S. Liu, A. J. Carty, S.-M. Peng, and G.-H. Lee, J. Mater. Res. 13, 1999 (2003).

${ }^{11}$ T. Aaltonen, P. Alén, M. Ritala, and M. Leskelä, Chem. Vap. Deposition 9, 45 (2003).

${ }^{12}$ O.-K. Kwon, J.-H. Kim, H.-S. Park, and S.-W. Kang, J. Electrochem. Soc. 151, G109 (2004).

${ }^{13}$ T. Aaltonen, M. Ritala, K. Arstila, J. Keinonen, and M. Leskelä, Chem. Vap. Deposition 10, 215 (2004).

${ }^{14}$ Y.-S. Min, E. J. Bae, K. S. Jeong, Y. J. Cho, J.-H. Lee, W. B. Choi, and G.-S. Park, Adv. Mater. 15, 1019 (2003).

${ }^{15}$ O.-K. Kwon, S.-H. Kwon, H.-S. Park and S.-W. Kang, Electrochem. Solid State Lett. 7, C46 (2004)

${ }^{16}$ S. M. Rossnagel and T. S. Kuan, J. Vac. Sci. Technol. B 22, 240 (2004).

${ }^{17}$ R. G. Gordon, D. Hausmann, E. Kim and J. Shepard, Chem. Vap. Deposition 9, 73 (2003).

${ }^{18}$ R. Wang, H. Chung,X. Tang, J. Y. Wang, W. D. Wang, Y. Tanaka, J. M. Yu and P. Gopalraja, US Patent Application US2007/0059502.

${ }^{19}$ Huazhi Li, Titta Aaltonen, Zhengwen Li, Booyong S. Lim, and Roy G. Gordon, to be published.

${ }^{20}$ J. S. Becker, S. Suh and R. G. Gordon, Chem. Mater. 15, 2969 (2003).

${ }^{21}$ J. J. Vlassak, Y. Lin and T.Y. Tsui, Materials Science and Engineering A 391, 159 (2005). 


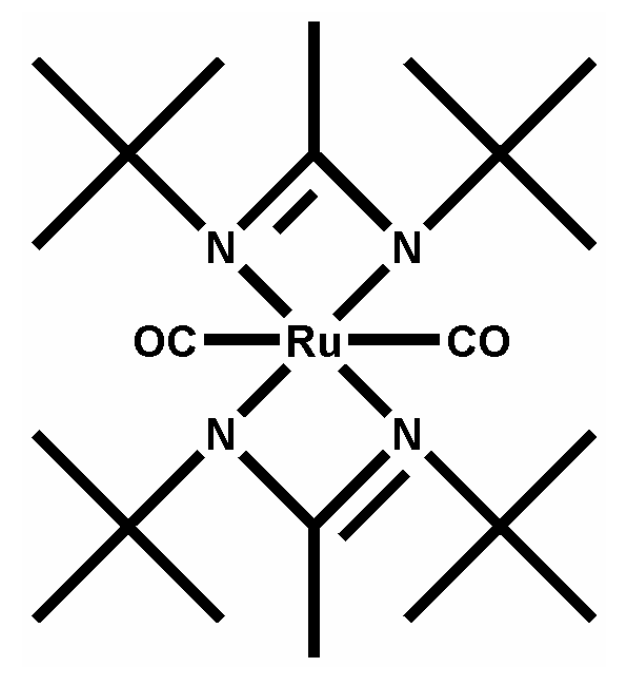

(a)

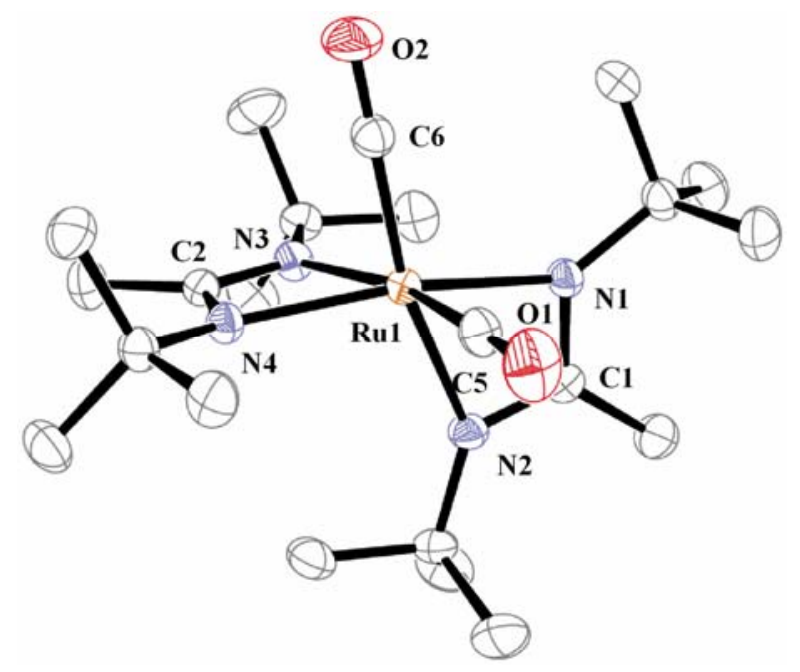

(b)

Figure 1. (a) The chemical formula of Ru precursor bis( $N, N^{\prime}$-di-tertbutylacetamidinato)ruthenium(II) dicarbonyl, Ru( $\left.{ }^{\mathrm{B}} \mathrm{Bu}-\mathrm{Me}-\mathrm{amd}\right)_{2}(\mathrm{CO})_{2}$.

(b) its molecular structure by X-ray crystallography (hydrogen atoms not shown). 


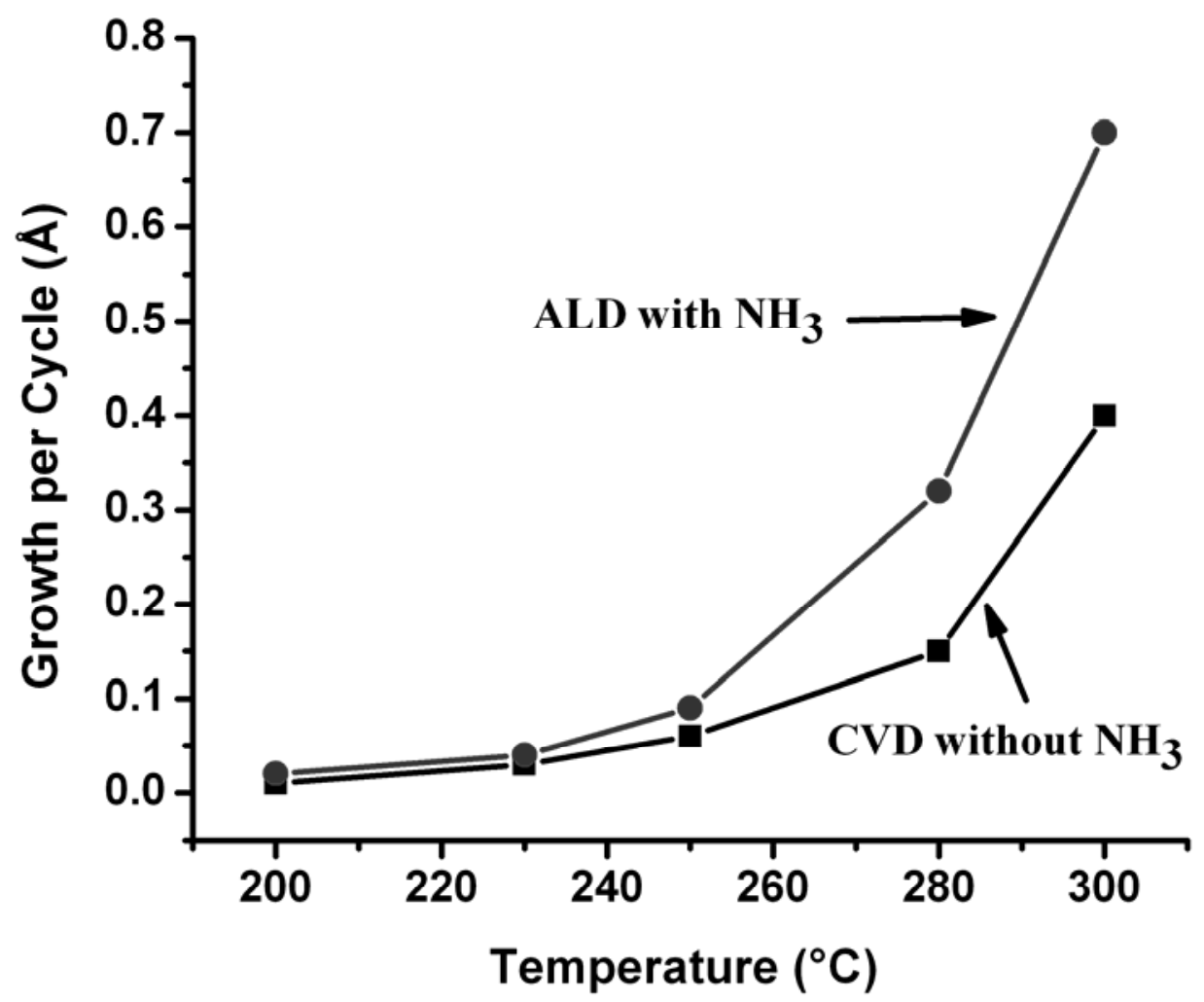

Figure 2. The Ru growth rate with $\mathrm{NH}_{3}(\mathrm{ALD})$ or without $\mathrm{NH}_{3}$ (pulsed CVD) at different temperatures. 


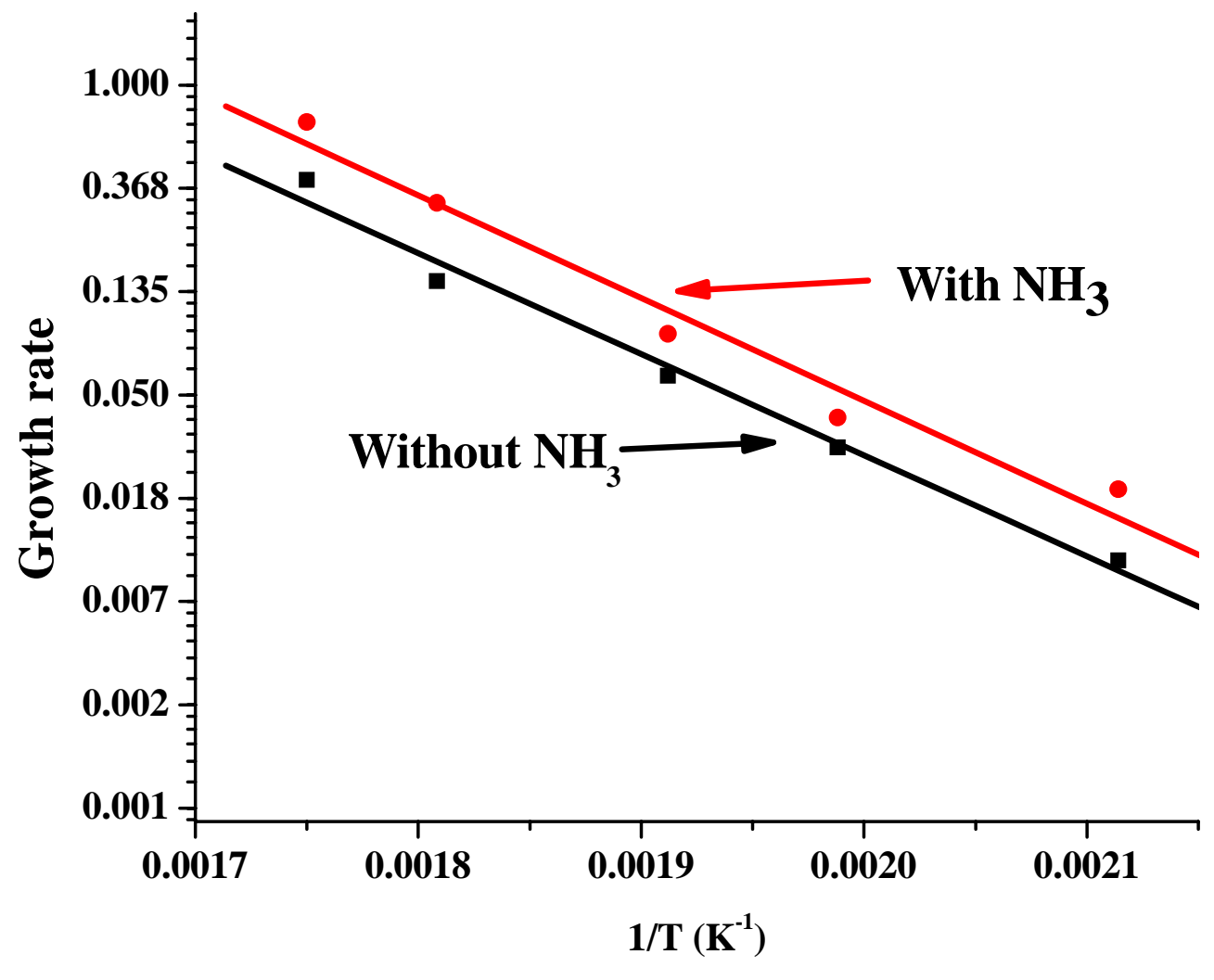

Figure 3. Pulsed CVD growth rate of Ru without using $\mathrm{NH}_{3}$ (squares) and ALD growth rate (circles) as a function of temperature. 


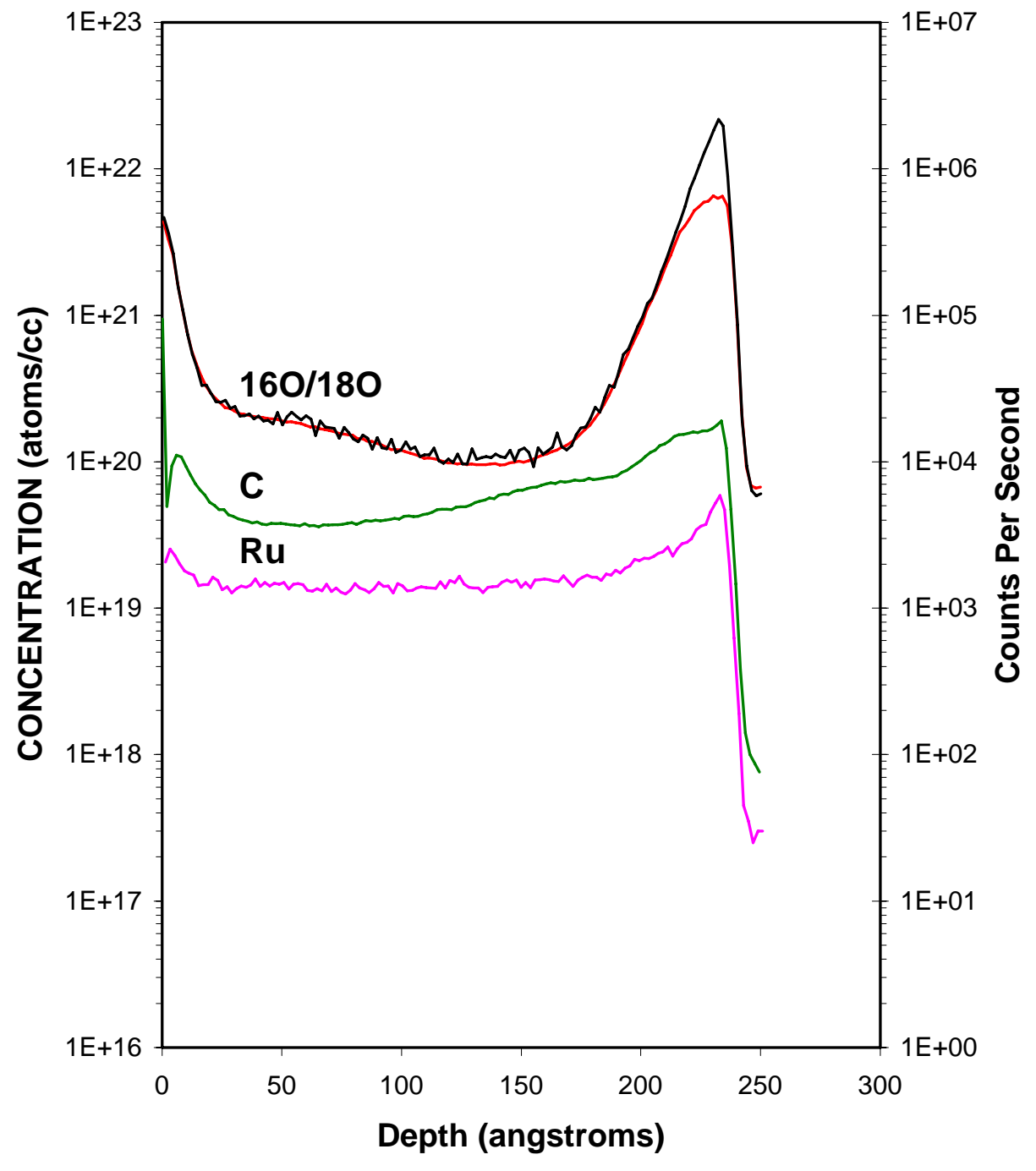

Figure 4. SIMS depth profiles of a ruthenium film $20 \mathrm{~nm}$ thick deposited on thermal $\mathrm{SiO}_{2}$ at $300{ }^{\circ} \mathrm{C}$; the observed carbon and oxygen counts/second have been converted to concentrations; the ruthenium curve represents the signal in counts per second because a sensitivity factor is not known for Ru. 


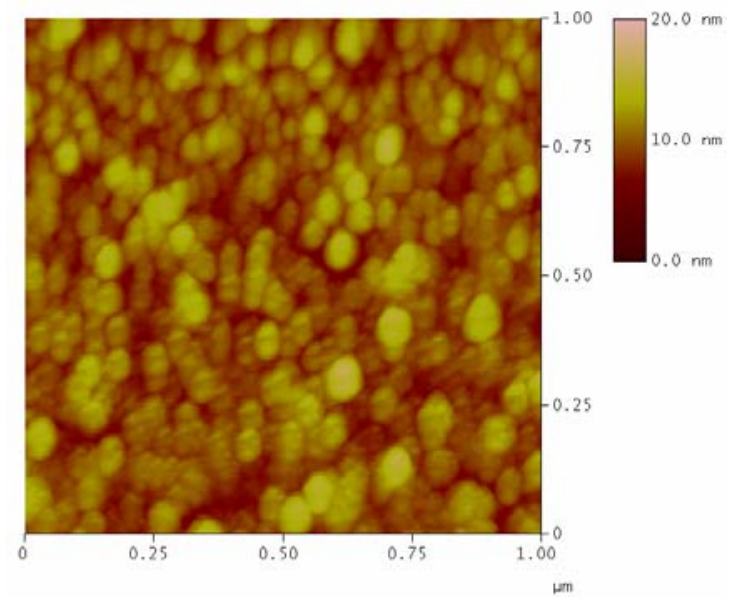

(a)

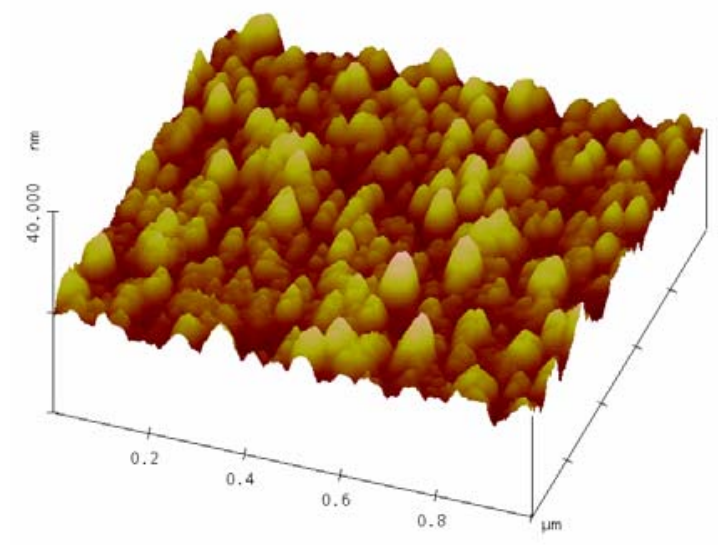

(b)

Figure 5. AFM image of an $87 \mathrm{~nm}$ ruthenium film grown at $300^{\circ} \mathrm{C}$. (a) 2-D view; (b) 3-D view. 


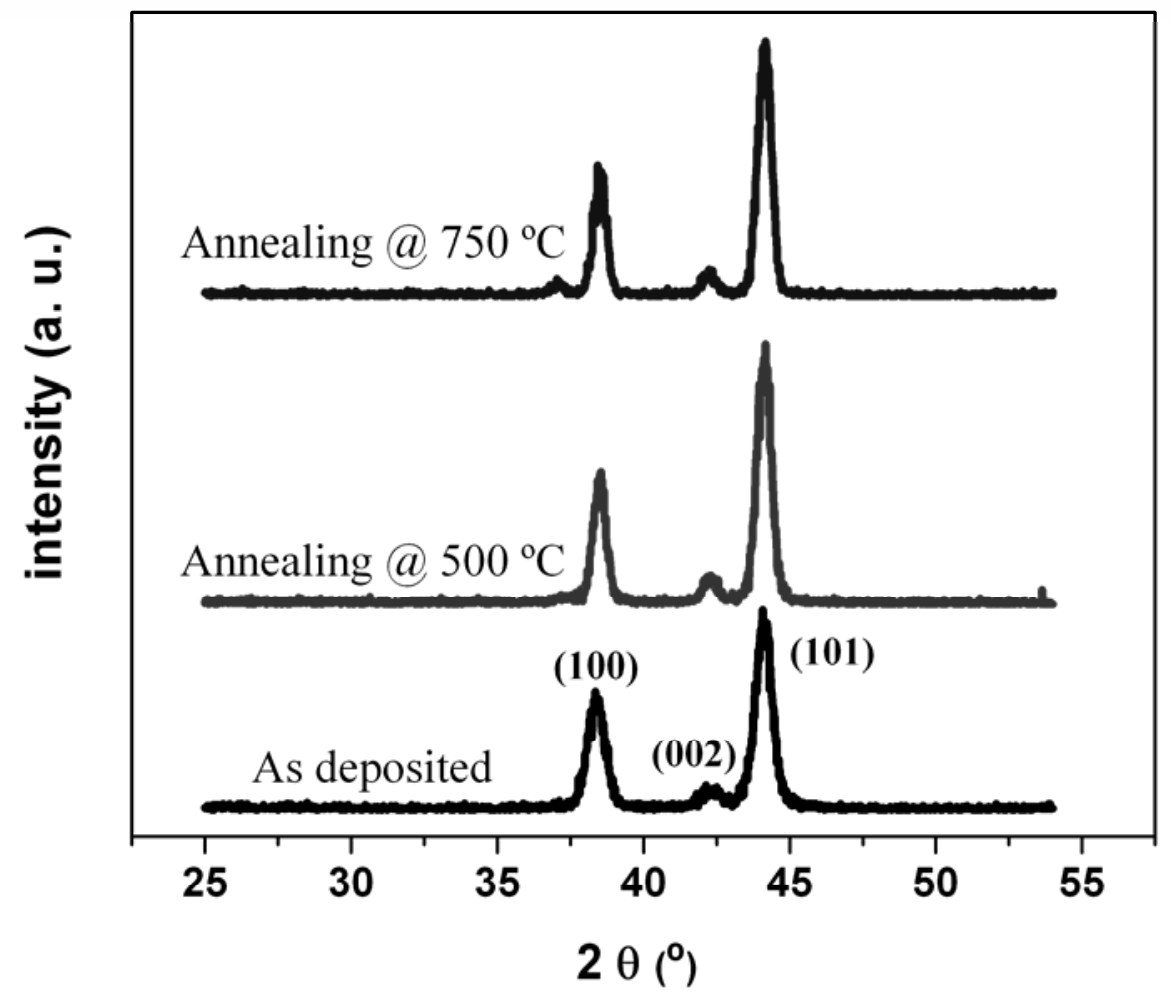

Figure 6. XRD patterns obtained from a Ru film as deposited and after annealing. 


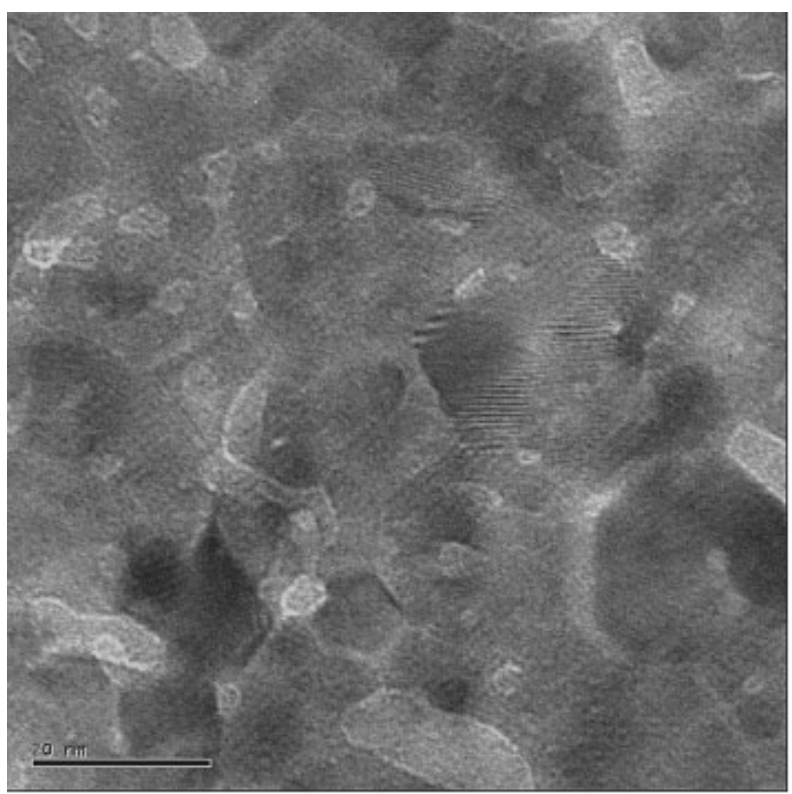

$20 \mathrm{~nm}$

Figure 7. TEM images of a Ru film (30 nm) deposited over $\mathrm{Si}_{3} \mathrm{~N}_{4}$ membrane (30nm). 


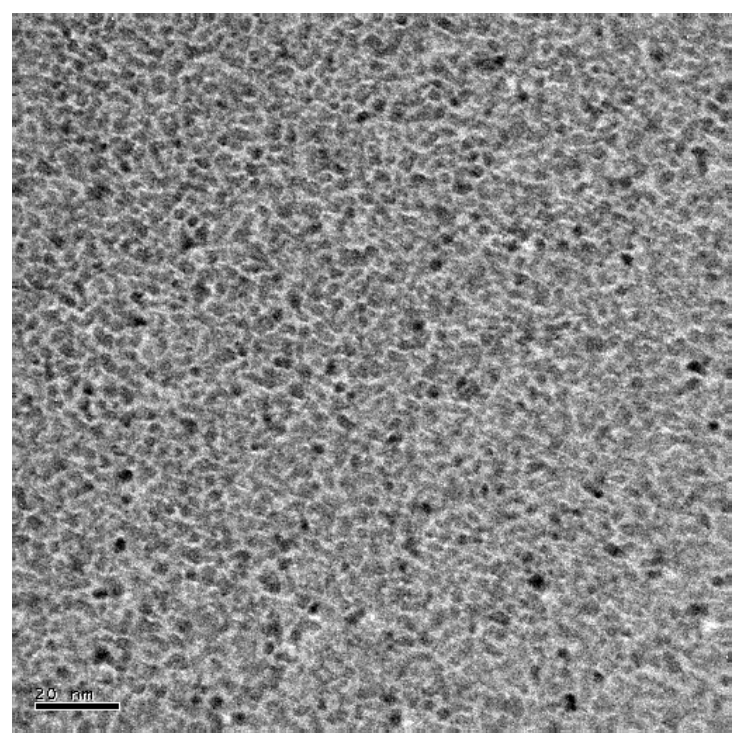

Figure 8. TEM of a Ru film grown on freshly deposited tungsten nitride on a $\mathrm{Si}_{3} \mathrm{~N}_{4} \mathrm{TEM}$ membrane. 


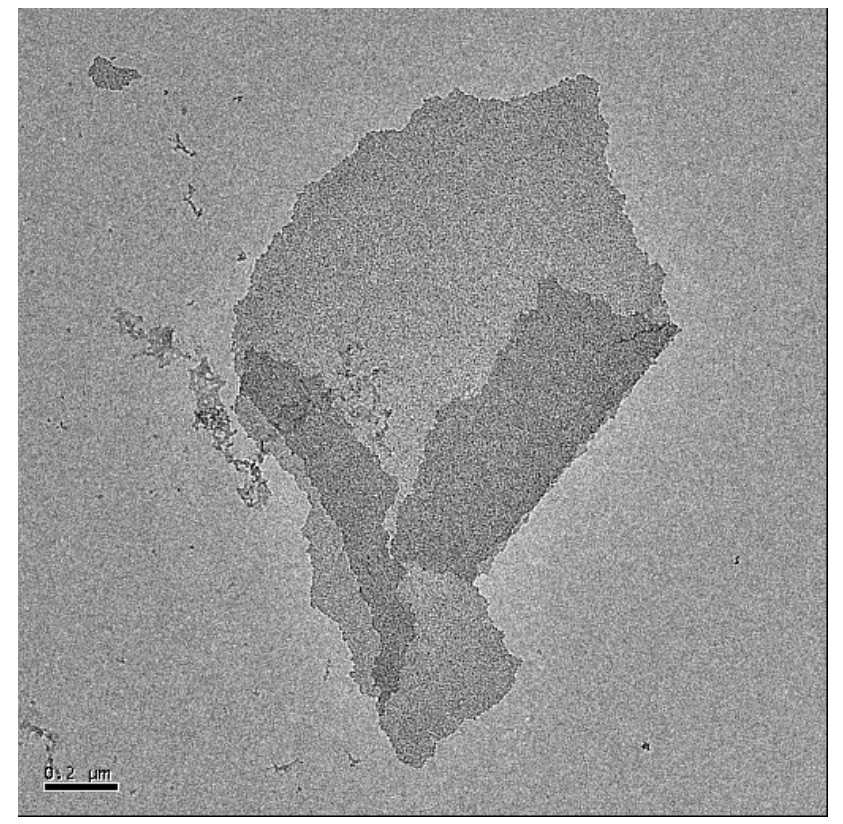

Figure 9. TEM image of a fragment of a Ru film of after etching of WN in a pierced film of $\mathrm{Ru}(2 \mathrm{~nm}) / \mathrm{WN}(3 \mathrm{~nm}) / \mathrm{SiO}_{2}$ deposited on $\mathrm{Si}_{3} \mathrm{~N}_{4}$ membrane $(30 \mathrm{~nm})$. The darker areas are regions where the film folded over on itself. 


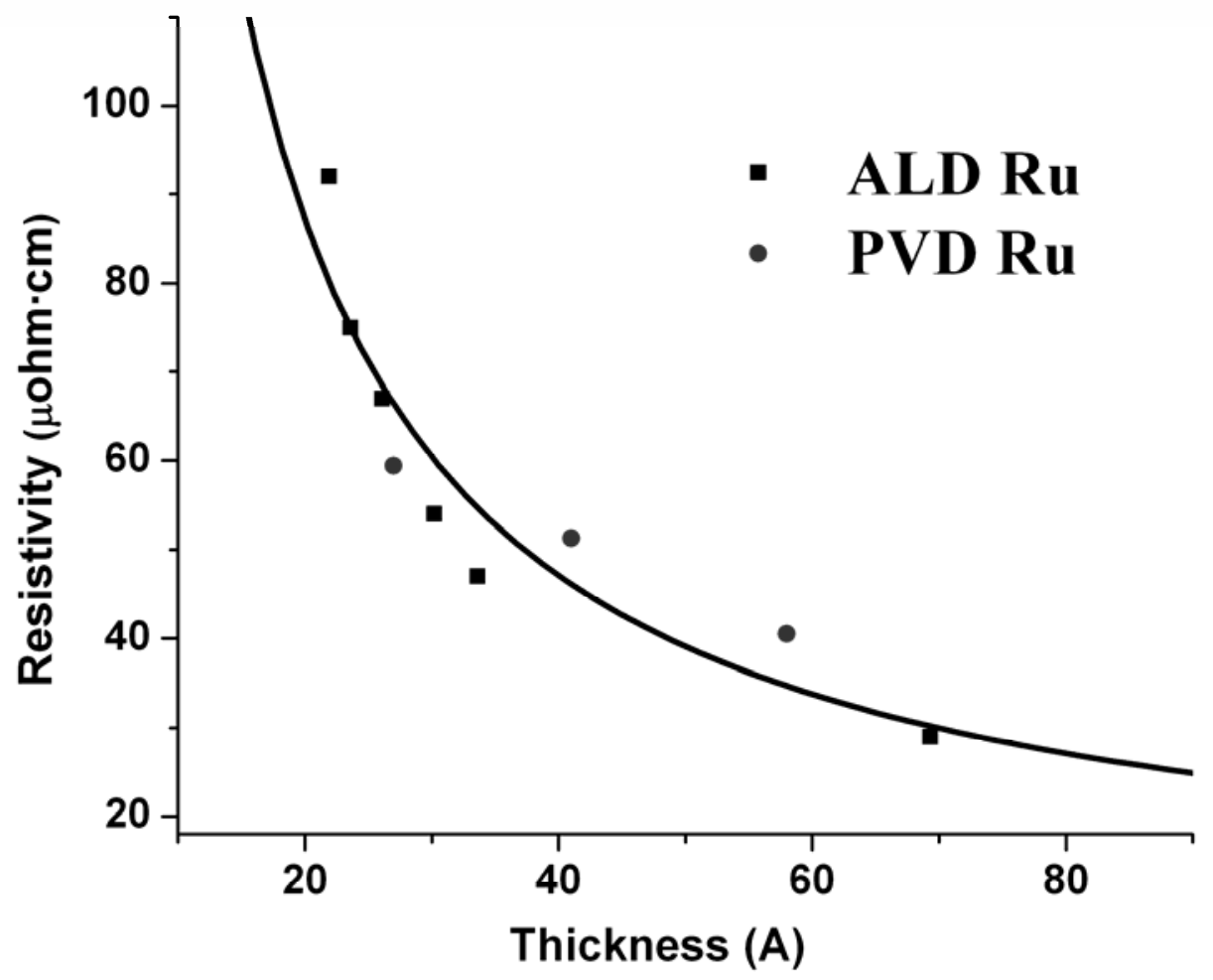

Figure 10. The resistivity of Ru thin films as a function of Ru film thickness on WN substrates. Black squares are data from this study, gray dots are data from sputtered Ru. 


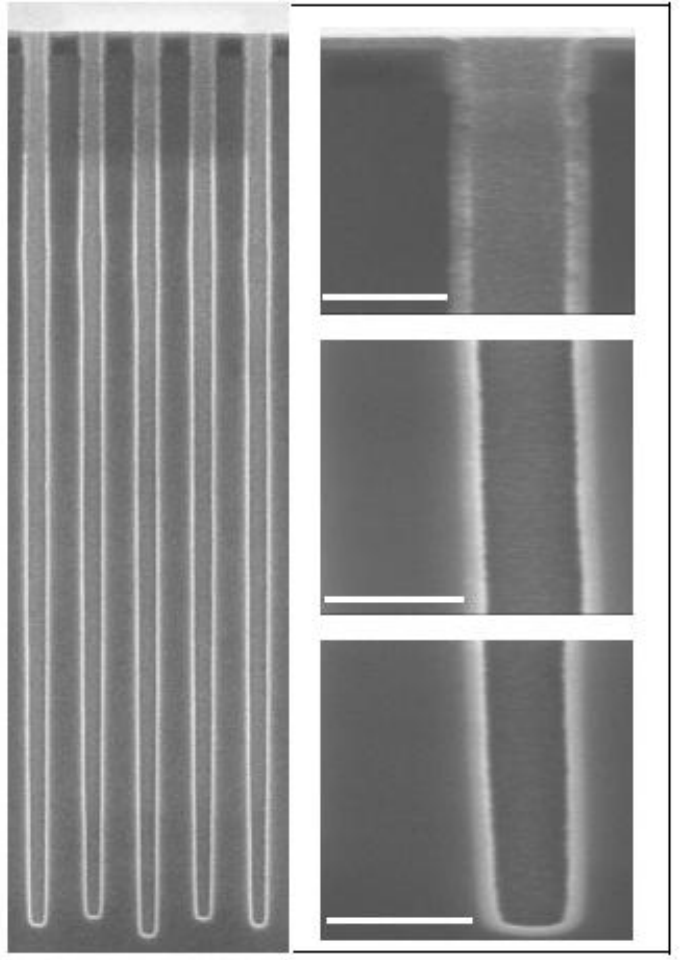

Figure 11. Ru films deposited inside the high aspect ratio (40:1) trench structure showing excellent conformality of pulsed CVD process. The white bar on the bottom of each image represents $200 \mathrm{~nm}$. 